Trauma Berufskrankh 2009 · 11 [Suppl 2] 160-170

DOI 10.1007/s10039-008-1471-0

Online publiziert: 7. Februar 2009

(c) Springer Medizin Verlag 2009
F. Bonnaire - T. Lein · K.-J. Engler · F. Moritz

Klinik für Unfall- Wiederherstellungs- und Handchirurgie,

Städtisches Klinikum, Krankenhaus Dresden Friedrichstadt

\section{Arthroskopisch gestützte Rekonstruktion von Tibiakopffrakturen}

\section{Sinnvolle Indikationen und Technik}

Tibiakopffrakturen mit Gelenkbeteiligung und Frakturdislokation werden seit Jahrzehnten mittels offener Operationstechnik mit den Prinzipien

- Reposition der Gelenkflächen,

- Wiederherstellung des Gelenksockels,

- Rekonstruktion der Bandstrukturen und Menisken und

- nachfolgender Osteosynthese

behandelt. Hauptproblem ist der v. a. bei komplexeren Verletzungen immer vorhandene, unfallbedingte Weichteilschaden, der bei den operativen Maßnahmen zusätzlich verstärkt werden kann. Der Wunsch nach Vermeidung einer zusätzlichen Traumatisierung des Weichteilmantels und einer gleichzeitigen sicheren und optisch kontrollierten Rekonstruktion des Gelenks führte zur arthroskopisch gestützten Operation. Harper et al. [9] gaben seit 1979 perkutane Repositionstechniken mit nachfolgender Schraubenosteosynthese am Tibiakopf an $[9,14]$.

Mit der Weiterentwicklung der Arthroskopie zur Diagnostik und Therapie von Kniebinnenschäden kam bei Unfallchirurgen und Orthopäden in Deutschland und Europa das Interesse auf, auch bestimmte Frakturen des Tibiakopfs minimalinvasiv arthroskopisch gestützt oder gedeckt zu versorgen. Nachdem Mitte der 1980er Jahre zunächst die endoskopische Kontrolle der Frakturreposition angestrebt wurde, entwickelte sich die arthroskopisch-radiologisch gestützte Versorgung bestimmter Frakturtypen gegen Ende der 1980er Jahre in vielen Zentren zum Routineeingriff $[4,7,10,15,22,25]$. Der anfänglichen Euphorie und dem breiten Interesse an solchen Verfahren folgte eine Lernkurve, welche nicht frei war von Misserfolgen. Eine Differenzierung der Indikationen für bestimmte Typen von Tibiakopffrakturen war eine notwendige Folge.

\section{Klassifikation der Tibiakopffrakturen}

Je nach Qualität des Knochens, dem Mechanismus und der Rasanz des Traumas resultieren durch die Krafteinwirkung am Schienbeinkopf

- Spalt- oder Impressionsfrakturen,

- Trümmerfrakturen des Tibiaplateaus oder aber

- Luxationsfrakturen mit z. T. ausgedehnten ligamentären Verletzungen bei radiologisch eher dezenter knöcherner Mitbeteiligung am Rand des Tibiaplateaus („rim fracture medial“, Ségond-Fragment lateral).

Hinsichtlich ihrer Behandlung und Prognose können Tibiakopffrakturen nach morphologischen und funktionellen $\mathrm{Ge}$ sichtspunkten wie folgt unterteilt werden:

\section{Tibiaplateaufrakturen}

Sie resultieren meist aus einer axialen Krafteinwirkung und führen zu einer Spaltung und/oder Impression des Tibia- plateaus. Ligamentäre oder neurovaskuläre Begleitverletzungen sind eher selten. Als Einteilung empfiehlt sich die AOKlassifikation 41A1-C3 (• Abb. 1, [19]).

\section{AO-Klassifikation}

Typ-A-Frakturen. Es handelt sich um Brüche ohne Beteiligung der artikulierenden Gelenkfläche.

Die Eminentiafrakturen werden zu dieser Gruppe subsumiert und als A1 klassifiziert. A2-Frakturen sind einfach extraartikulär, Typ-A3-Frakturen gehen immer mit Hochrasanztraumen und einem schweren Weichteilschaden einher. Das Gelenkplateau ist bei diesen Brüchen intakt.

Typ-B-Frakturen. Sie stellen einfache Gelenkfrakturen mit Beteiligung nur eines Tibiagelenkflächenplateaus dar. Typ B1 beinhaltet Spaltfrakturen, Typ-B2-Verletzungen sind Impressionsfrakturen und bei Typ B3 handelt es sich um die Spaltund Impressionsfrakturen.

Typ-C-Frakturen. Sie sind grundsätzlich bikondylär und in ihrer Bruchform eher einfach (Typ C1), mit mehr oder weniger ausgeprägter metaphysärer Beteiligung (Typ C2) oder mit zusätzlichen Impressionen auf Gelenkniveau (Typ $\mathrm{C}_{3}$ ).

\section{Klassifikation nach Schatzker}

Sie stellt eine weitere - durchaus etablierte - Einteilung dar. Sie zielt ebenfalls auf morphologische Kriterien ab und un- 
terscheidet 6 Frakturtypen (• Abb. 2, [26]):

I. reiner Spaltbruch

II. Spaltimpressionsbruch

III.isolierte Impressionsfraktur

IV. Fraktur des medialen Kondylus mit Eminentiafraktur

V. bikondyläre Fraktur

VI.intraartikuläre Fraktur mit meta- und diaphysärer Dissoziation (Luxationsfraktur)

\section{Luxationsfrakturen}

Sie entstehen v. a. durch Flexions-, Rotations- und Distraktionsmechanismen am Kniegelenk. Sie werden nach der MooreKlassifikation eingeteilt ( $\bullet$ Abb. 3):

- Typ I („split“)

- Typ II („entire condyle“)

- Typ III („rim avulsion“)

- Typ IV („rim compression2)

- Typ V („four part“)

Typ I nach Moore betrifft ausschließlich das mediale Plateau mit einem häufig nach dorsal verschobenen Fragment. Die Frakturlinie verläuft beim Typ II in das kontralaterale Kompartiment hinein und umfasst auch die Interkondylärregion mit den Kreuzbandverankerungen. Die als „Ségond-Fraktur“ oder laterales Kapselzeichen bezeichnete Typ-III-Fraktur entspricht einem knöchernen Ausriss des lateralen Kapsel-Band-Apparats mit komplexer Instabilität und Beteiligung der Kreuzbänder. Beim Typ IV resultiert die Impaktion eines unter dem Meniskus liegenden Kantenfragments. Die 4-Fragment-Fraktur (Typ V) hat eine hohe Inzidenz für neurovaskuläre $\mathrm{Be}$ gleitverletzungen und ist nicht selten mit einer Fraktur des Fibulaköpfchens assoziiert. Im Gegensatz zur bikondylären Tibiaplateaufraktur ist hier der zentrale $\mathrm{Ge}$ lenkpfeiler mit der Eminentia intercondylaris zusätzlich ausgerissen.

\section{Frakturen im Wachstumsalter}

Am Tibiakopf treten alterspezifische Epiphysenlösungen und -frakturen relativ selten auf, sie können nach Salter u. Harris [26] oder Aitken $[1,2]$ eingeteilt werden. Typische Apophysenverletzungen (Lig. patellae) finden eine eigene Beschreibung bei
Trauma Berufskrankh 2009 · 11 [Suppl 2]: 160-170 DOI 10.1007/s10039-008-1471-0

(c) Springer Medizin Verlag 2009

\section{F. Bonnaire $\cdot$ T. Lein $\cdot$ K.-J. Engler $\cdot$ F. Moritz}

Arthroskopisch gestützte Rekonstruktion von Tibiakopffrakturen. Sinnvolle Indikationen und Technik

\section{Zusammenfassung}

Die arthroskopisch unterstützte Osteosyn-

these von Tibiakopffrakturen ist indiziert bei Eminentiafrakturen von Kindern sowie bei Erwachsenen mit isoliert auftretenden Brüchen. Weitere gute Indikationen werden von uns bei den 41B1-3-Frakturen nach AO-Klassifikation und bei den Avulsionsfrakturen gesehen. Die arthroskopische Technik ist aufwändig, zeitraubend und anspruchsvoll. Zusatzinstrumente wie kanülierte Stößel, Zielgeräte und Repositionshilfen sowie eine gut geplante Operation mit intraoperativer Fluoroskopie sind obligat. Bei Durchführung des Eingriffs durch erfahrene Arthroskopeure wurden durchweg sehr gute und gute Ergebnisse in $>90 \%$ der Fälle mitgeteilt. Komplikationen wurden selten beschrieben. Die

Methode verbindet die Chancen einer schonenden Reposition unter Sicht mit der Beurteilung und Mitbehandlung der gesamten Gelenkstrukturen einschließlich Meniskus, Band- und Knorpelverletzungen und einer gezielten, effektiven und minimalinvasiven Osteosynthese. Speziell bei Vorerkrankungen und Begleitverletzungen ist sie für die Beurteilung des Unfallzusammenhangs und der festgestellten Veränderungen aufgrund der Visualisierungs- und Dokumentationsmöglichkeiten sehr hilfreich.

\section{Schlüsselwörter}

Tibiakopffraktur · Arthroskopisch unterstützte Osteosynthese - Zusatzinstrumentarium . Visualisierung · Dokumentation

\section{Arthroscopy-assisted reconstruction of tibial plateau fractures. Reasonable indications and technique}

\section{Abstract}

Arthroscopy-assisted osteosynthesis of tibial plateau fracture is indicated in eminence fractures in children and adults with isolated fractures. We also consider 41B1-3 fractures according to the $\mathrm{AO}$ classification and avulsion fractures to be strong indications. The arthroscopic technique is complex, time-consuming and challenging. Additional instruments, such as cannulated pestles, targeting devices and reduction tools, as well as a carefully planned surgical intervention with intraoperative fluoroscopy are mandatory. When performed by an experienced arthroscopist, good to very good results are reported in $90 \%$ of cases. Complications are rare. The method combines the opportunity for gentle reduction under vision with assessment and simultaneous treatment of all joint structures, including meniscal, ligament and cartilage injuries, as well as targeted, effective and minimally invasive osteosynthesis. The technique is very helpful in assessing the method of accident and diagnosed changes, particularly in the case of previous disease and accompanying injuries, due to good visualisation and documentation.

\section{Keywords}

Tibial plateau fracture - Arthroscopy-assisted osteosynthesis · Additional instruments . Visualisation · Documentation 


\section{Knieverletzungen}

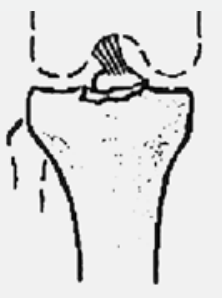

A1

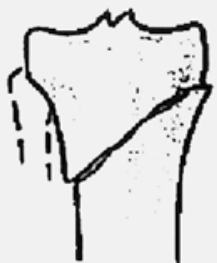

A2

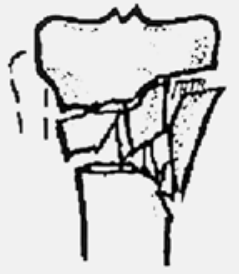

A3

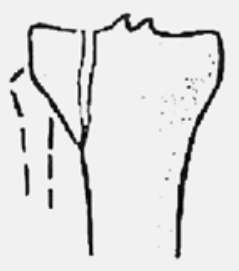

B1

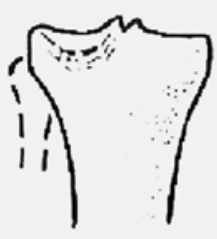

B2

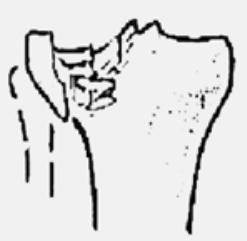

B3

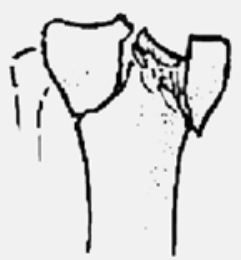

C3

Abb. $1 \Delta$ AO-Klassifikation der Tibiakopffrakturen: Typ A extraartikulär, Typ B unikondylär, Typ C bikondylär, Erläuterungen s. Text. (Nach [18])

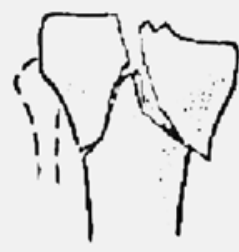

C1

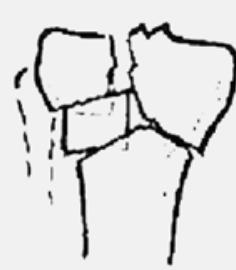

c2

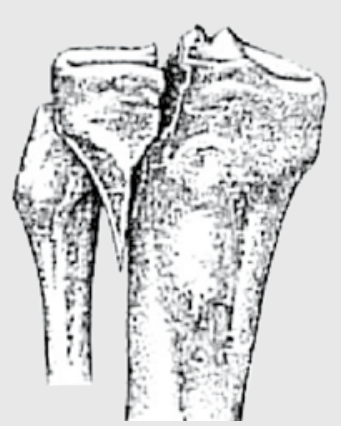

Typ I

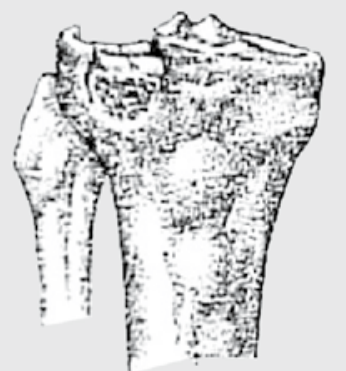

Typ III

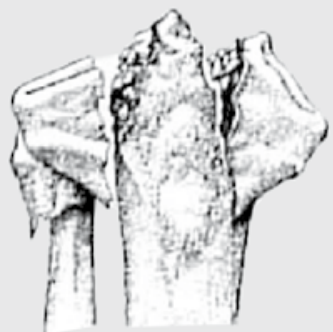

Typ V

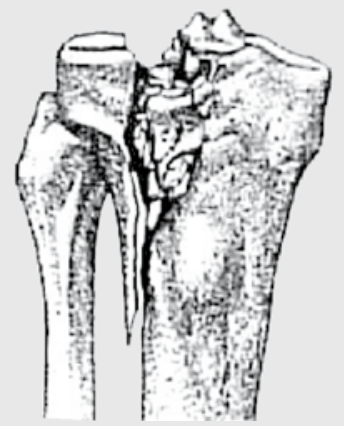

Typ II

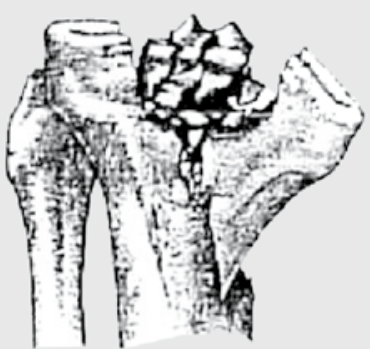

Typ IV

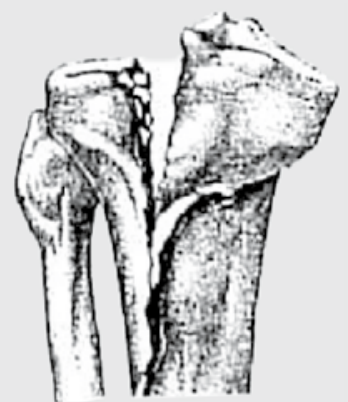

Typ VI

Abb. $2 \Delta$ Klassifikation nach Schatzker, Erläuterungen s. Text

Watson-Jones [31]. Eine Besonderheit stellen die knöchernen Abrissverletzungen der Eminentia intercondylaris mit Ausriss der Kreuzbandverankerung dar, die Einteilung erfolgt hier nach den Kriterien von Meyers u. McKeever (• Abb. 4, [17]):

- Typ I: Fissur in der vorderen Eminentia (Eminentia geringfügig angehoben)

- Typ II: Anhebung des Fragments bis zur Hälfte seiner Ausdehnung, hinterer Anteil fest

- Typ III: vollständiges Lösen der Eminentia intercondylaris

- Typ IV: zusätzliche Rotation des Fragments.

\section{Diagnose}

\section{Klinische Untersuchung}

Auffällig sind die Schwellung der Kniegelenkregion mit schmerzhafter Funktionseinschränkung und je nach Alter der Verletzung ein Weichteilhämatom, ein eindeutiger Erguss kann fehlen. Die Symptomatik ist je nach Verletzungsausmaß sehr variabel, eine initiale Luxation am Kniegelenk kann zwischenzeitlich reponiert sein und leicht übersehen werden. Die Stabilitätsprüfung des verletzten Kniegelenks mag initial wegen Schmerzen und Schwellung nicht eindeutig zur Befundklärung beitragen, sollte aber unter der gebotenen Vorsicht nicht unterlassen werden.
Neben der Prüfung auf Bandinstabilität und Kniegelenkführung müssen Pulsstatus und periphere sensible und motorische Defizite erfasst werden. Da arterielle Verletzungen in etwa $0,7 \%$ der Fälle bei Tibiakopffrakturen auftreten, müssen im Zweifelsfall eine dopplersonographische Untersuchung oder eine Angiographie angeschlossen werden [11]. Sensomotorische Defizite können Hinweise auf eine Affektion des N. peronaeus sein (hohe Fibulafraktur!), sind jedoch auch nicht selten durch ein drohendes oder manifestes Kompartimentsyndrom bedingt. Bei Verdacht kann eine Kompartimentdruckmessung zur Klärung führen. Bei Unklarheiten kann man nur zur Spaltung der Kompartimente raten, denn die Häufigkeit eines begleitenden Komparti- 


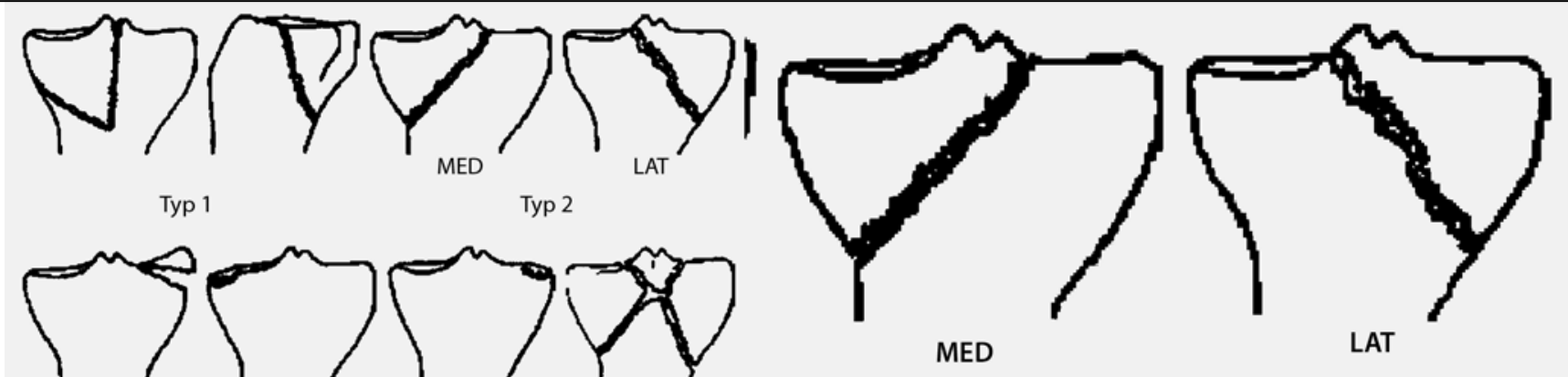

Typ 2

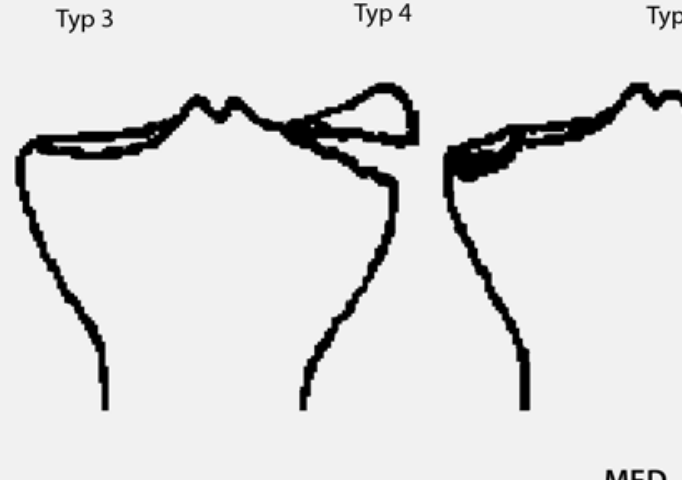

Typ 3
MED
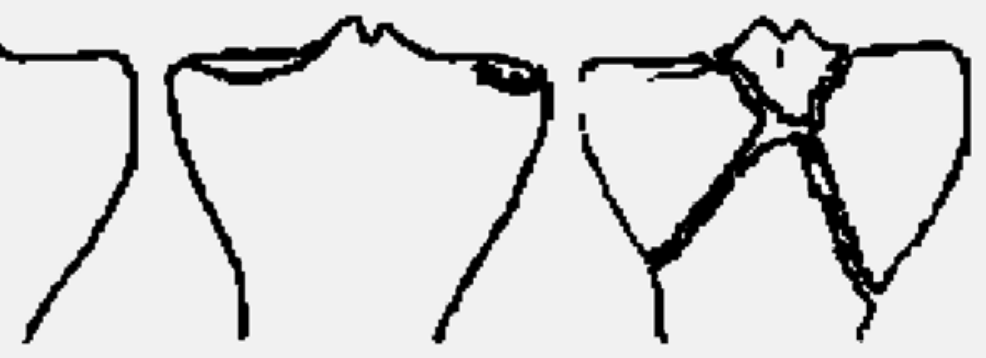

LAT

Typ 4

Typ 5

Abb. $3 \Delta$ Klassifikation nach Moore, LAT lateral, MED medial, Erläuterungen s. Text

mentsyndroms bei $\mathrm{AO}_{41} \mathrm{~B}$ - und -C-Tibiakopffrakturen wird mit $5-10 \%$ angegeben [28].

\section{Apparative Diagnostik}

Als radiologische Basisdiagnostik sind die Aufnahme des Kniegelenks in 2 Ebenen (Zentralstrahl auf den Gelenkspalt) und der Patella tangential Standard. Bei Verdacht auf eine Tibiakopfimpressionsfraktur kann dann ggf. eine Plateauaufnahme mit in a.-p. Strahlengang $10^{\circ}$ kraniokaudal gekippter Röntgenröhre angeschlossen werden.

Weitere Spezialaufnahmen sind in der Regel entbehrlich, da bei Frakturen mit Gelenkbeteiligung eine Spiral-CT-Untersuchung mit multiplanarer Darstellung und $3 \mathrm{D}$-Rekonstruktion zu fordern ist, denn das Frakturausmaß wird in der Projektionsradiographie oft unterschätzt. Darüber hinaus ist eine subtilere Operationsplanung möglich (• Abb. 5).

Eine MRT-Untersuchung des Kniegelenks gehört nicht zur Routine in der Akutdiagnostik bei Tibiakopffraktur. Sie kann allenfalls bei speziellen Fragestellungen, z. B. bei Verdacht auf eine Ségond-

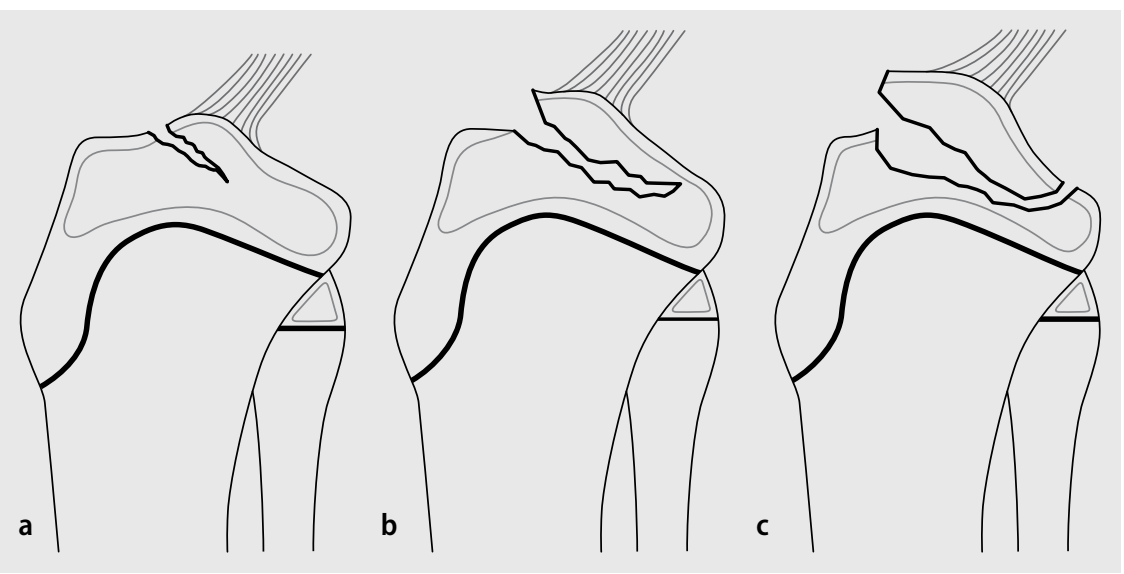

Abb. 4 \ Einteilung der Eminentiafrakturen nach Meyers u. McKeever, a Typ I, b Typ II, c Typ III. (Nach [17])

Fraktur, indiziert sein. Gelegentlich dient sie bei persistierenden Beschwerden und unauffälligem Röntgenbild dem Nachweis okkulter Frakturen oder Zonen mit „bone bruise“.

\section{Therapie}

\section{Konservative Therapie}

Nicht verschobene, stabile Brüche und kleine Impressionsfrakturen (Stufen bis
2 mm können toleriert werden [16]) können im Einzelfall konservativ versorgt werden. Die Behandlung besteht neben abschwellenden Maßnahmen in frühzeitiger Bewegung unter dem Schutz einer Orthese. Bewegungsschienen haben sich bewährt. Die konservative Behandlung mit Extension und Anmodellieren der Gelenkflächen durch geschlossene Reposition ist äußerst selten erforderlich [20]. Die Notwendigkeit einer frühen Bewegung im Kniegelenk zum Erhalt der Beweglichkeit 

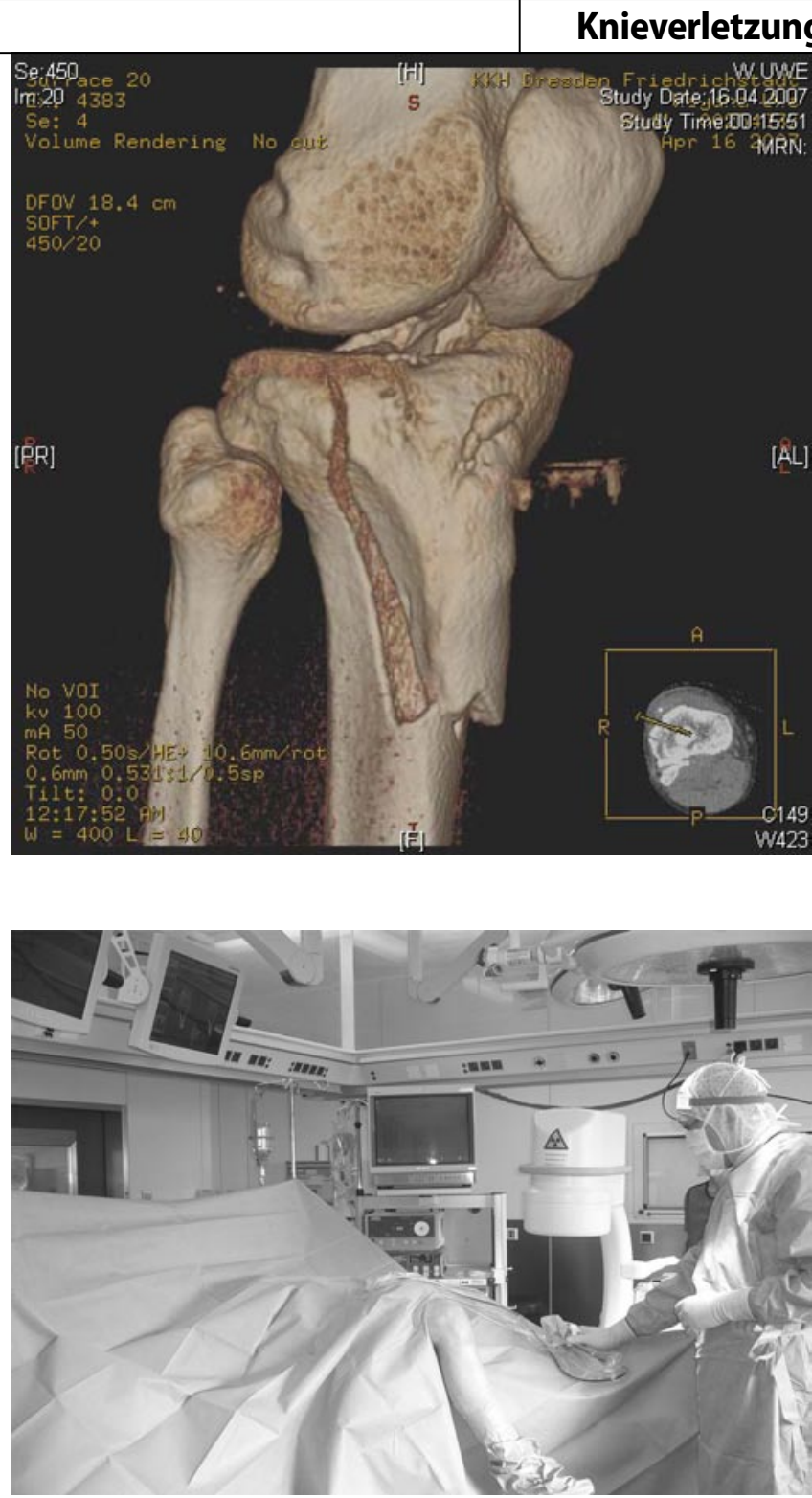

Abb. $5 \varangle$ Beispiel für eine 3D-Rekonstruktion bei Schienbeinkopfimpressionsspaltfraktur mit Eminentiabeteiligung zur präoperativen Planung des Vorgehens

Abb. $6<$ Intraoperatives Setting zur arthroskopisch und BVgestützten Versorgung bei Tibiakopffrakturen, BV Bildverstärker, weitere Erläuterung s. Text wurde v. a. von Tscherne [30] immer betont. Ein Fixateur externe zur vorübergehenden Stabilisierung ist immer möglich.

Teilweise gute Ergebnisse der konservativen Behandlung dürfen aber nicht darüber hinwegtäuschen, dass dieser Weg für die meisten Patienten durch die längere Immobilisationszeit und höhere Liegezeiten nicht zu akzeptieren ist.

\section{Operative Therapie}

\section{Planung einer arthroskopisch gestützten Operation bei Tibiakopffraktur}

Ziel ist eine operative Versorgung innerhalb der 1 . Woche nach dem Unfall. Einfluss auf den Zeitpunkt der Versorgung haben: um die Gruppe der Frakturen mit nur geringer knöcherner Beteiligung.

Nach Auswertung des Röntgenbildes und der Computertomographie - mit multiplanarer Rekonstruktion und 3D-Darstellung - sind komplexe, bikondyläre Luxations- oder Trümmerfrakturen besser offen zu operieren, da eine Verlängerung des Eingriffs durch den arthroskopischen $\mathrm{Zu}$ satz keinen adäquaten Zugewinn erwarten lässt und das Risiko für eine zusätzliche Weichteilbelastung zusätzlich erhöht. Der Eingriff kann mit und ohne Oberschenkelblutleere erfolgen. Präoperativ erfolgt die i.v. „single shot“-Prophylaxe mit einem Zephalosporin der 2. Generation.

C-Bogen und Bildverstärker werden auf der Seite des unverletzten Tibiaplateaus positioniert, der Arthroskopieturm ipsilateral. Die Kniegelenkregion sollte frei durchleuchtbar sein, das verletzte Bein wird mit $20^{\circ}$ Beugung in Hüft- und Kniegelenk gelagert, um die a.-p. Darstellung mit senkrechter Bildverstärkerposition (BV-Position) sowie die seitliche Durchleuchtung ohne zusätzliche Manipulationen zu ermöglichen. Das gesunde Bein muss in Hüfte und Knie gestreckt und etwas abgesenkt werden, alternativ kann es im gynäkologischen Beinhalter in Hüft- und Kniebeugung und Außenrotation der Hüfte gelagert werden. Wichtig sind der freie Zugang zum verletzten Gelenk und eine sicher Ablage der Arthroskopiekabel (• Abb. 6).

\section{Operationstechnik}

Zunächst erfolgt eine klinische Untersuchung in Narkose auf Bandinstabilitäten. Sie können mit Stressaufnahmen unter BVKontrolle dokumentiert werden. Über einen anterolateralen Standardzugang wird der Trokar eingeführt, das Hämarthros wird abgesaugt, und das Gelenk wird freigespült. Hilfreich zur Evakuation von Koageln und Fibrinfäden ist der Einsatz eines motorgetriebenen Synovialshavers. Beim sich anschließenden diagnostischen Rundgang durch das gesamte Gelenk werden die Kniebinnenstrukturen durch einen über den anteromedialen Arbeitszugang eingebrachten Tasthaken geprüft.

Eine Standardindikation zur arthroskopisch gestützten Versorgung sind die Eminentiaausrisse. Die Fraktur kann entweder mit dem Tasthaken oder über einen 

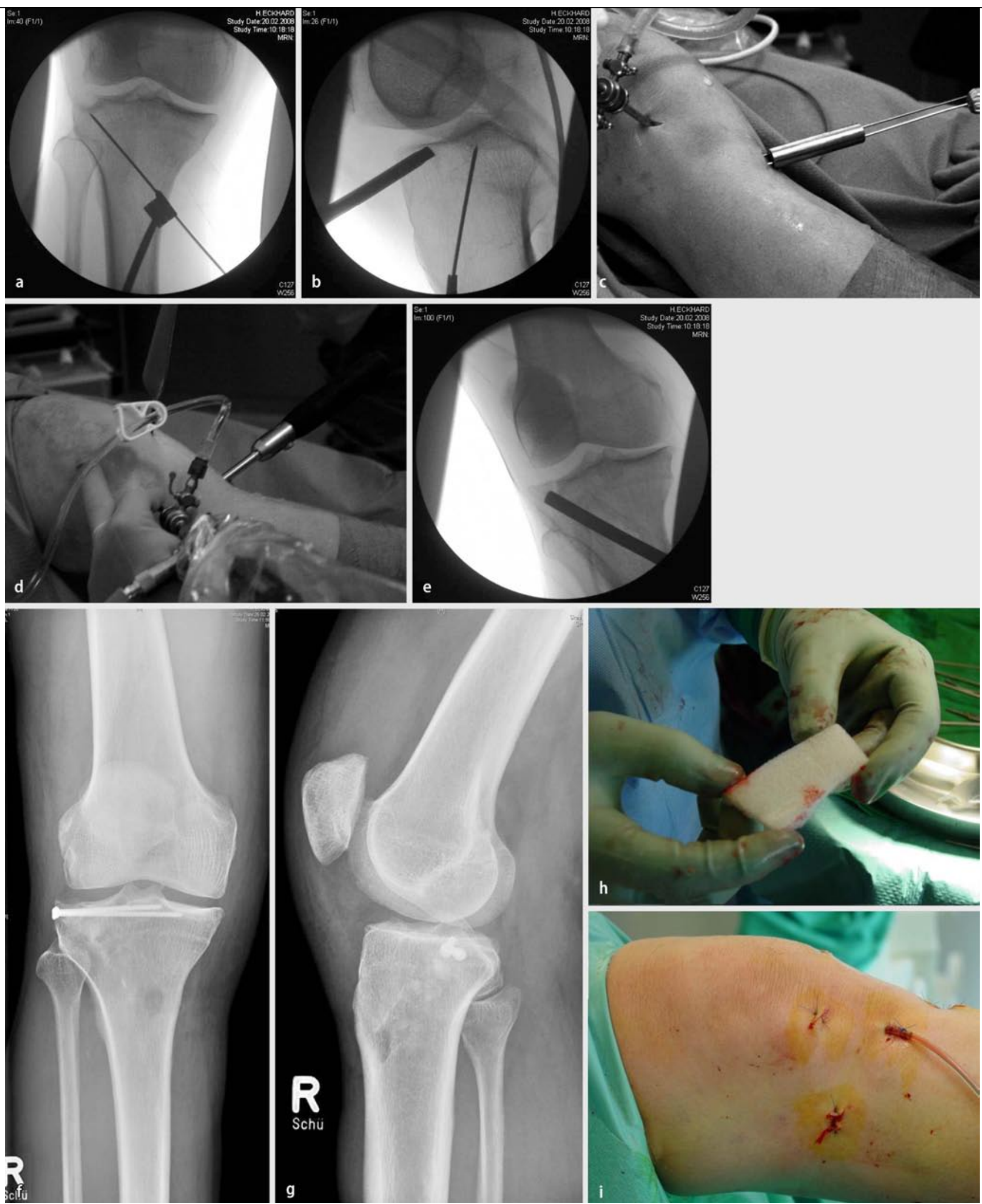

Abb. 7 \ Minimalinvasives Vorgehen mit Spezialinstrumenten aus dem Kreuzbandinstrumentarium, a Einbringen des Kirschner-Drahts von der Gegenseite (hier medial) unter BV-Kontrolle in 2 Ebenen, b-d bei nicht korrekter Lage (b) Möglichkeit des Einbringens eines neuen Drahts über das Zielgerät parallel an die korrekte Lokalisation, e Hochstößeln des Drahts über den kanülierten Stößel, $\mathbf{f , g}$ postoperative Röntgenbilder, $\mathbf{h}$ Lyostypt zum Auffüllen des Kanals, h,i Weichteile nach Verschraubung und Auffüllen des Kanals 

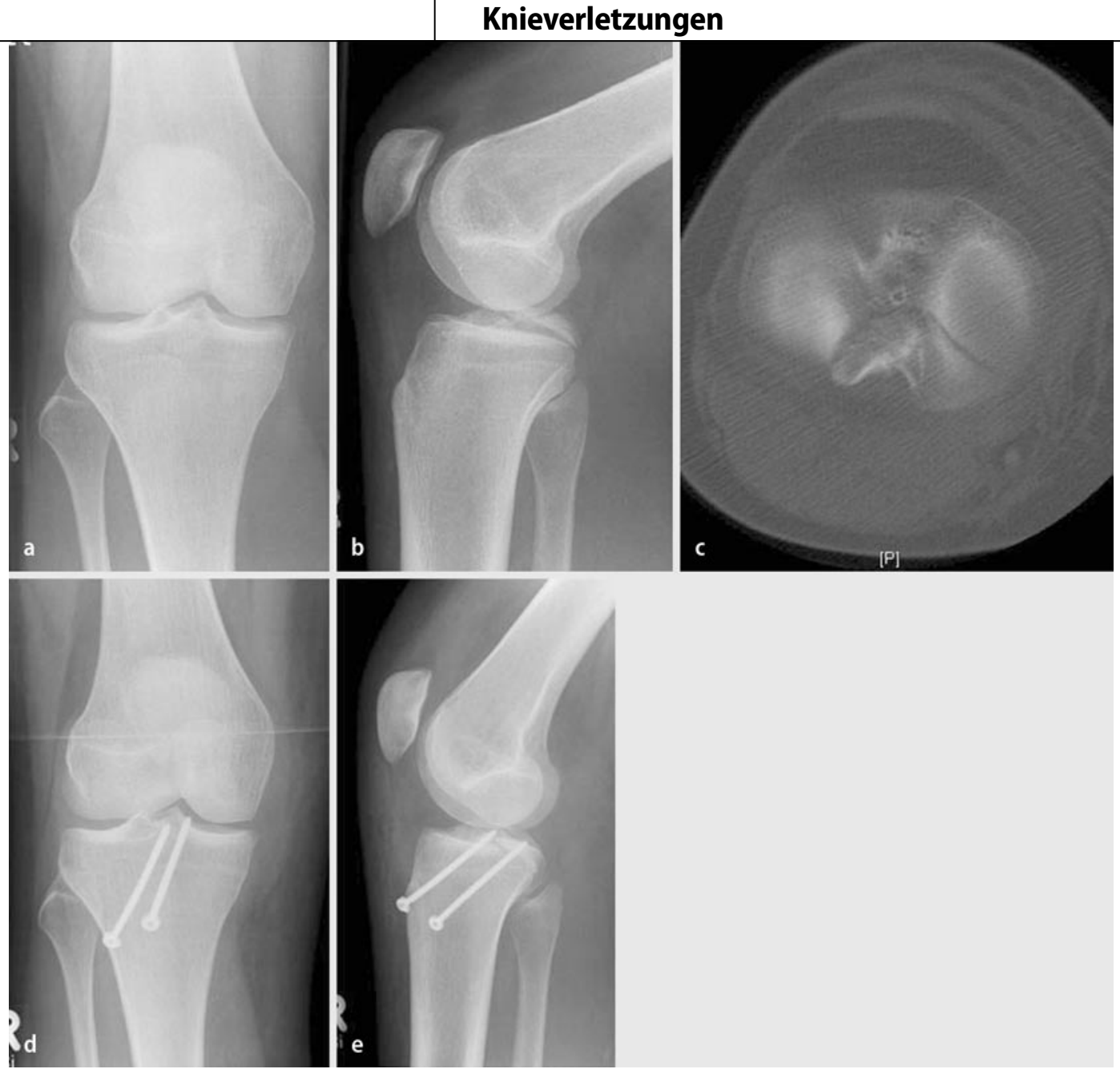

Abb. $8<$ Dorsal gelegene Eminentiafraktur bei 37-jährigem Sportler nach Hyperextensionstrauma, a,b Röntgenaufnahmen, c präoperativer CT-Scan zur Planung, d,e postoperatives Bild nach arthroskopisch gestützter Verschraubung

hohen anteromedialen Zugang mit einem Kirschner-Draht als Joystick reponiert werden. Dieses Manöver kann sich u. U. dann geschlossen schwierig gestalten, wenn das Lig. genu transversum in den Frakturspalt eingeklemmt ist oder wenn eine Fragmentation vorliegt. Kompromisse sollten bei der Reposition nicht geschlossen werden. Zur Not muss über eine Miniinzision offen reponiert werden.

Nach erfolgter Reposition können radiologisch kontrolliert Kirschner-Drähte, kanülierte Schrauben oder transossäre Nähte zur Fixation eingesetzt werden, wobei Instrumente aus dem Kreuzbandinstrumentarium sehr gut als Zielgeräte dienen können (• Abb. 7). Auch große, nicht zu stark dislozierte hintere Eminentiaausrisse können in Rückenlage mit dem kleinen Raspatorium reponiert und mit Zugschrauben fixiert werden (• Abb. 8a,b).

Seltene Begleitverletzungen an Menisken und Knorpel können arthroskopisch behandelt werden, sodass im Regelfall mit einer frühfunktionellen Nachbehandlung begonnen werden kann (• Abb. 9).

Bei den Tibiaplateaufrakturen sind es v. a. B-Frakturen, welche zu einer arthroskopisch unterstützten Frakturversorgung einladen, da sie am leichtesten und sichersten zu versorgen sind. Bei isolierten, mäßig dislozierten Spaltbrüchen (Typ ${ }_{41} \mathrm{~B} 1$ nach AO/Typ I nach Schatzker) genügt es, die imprimierte Komponente von der frakturierten Seite (in der Regel von anterolateral) über eine $2 \mathrm{~cm}$ lange Inzision mit einem Repositionsstößel anzuheben, arthroskopisch zu kontrollieren und dann perkutan unter radiologischer Kontrolle subchondral zu verschrauben, um die anatomische Breite des Tibiakopfs wiederherzustellen.

Impressionsfrakturen (• Abb. 9, 10) werden besser von der Gegenseite über ein Bohrloch an der medialen Kortikalis adressiert. Kannülierte Stößel können die Reposition erleichtern. Beim Vorbringen des Drahts ist immer eine Kontrolle mit dem Bildwandler in 2 Ebenen notwendig.
Beim Einbringen muss stets streng auf die Rotation des Kniegelenks und die Projektion des BV geachtet werden (• Abb. 9). Nach unserer Erfahrung reichen in der Regel gute subchondral platzierte KFI(K leinfragmentinstrumentarium)-Schrauben ausreichender Länge aus.

Die Stabilitätskriterien dürfen nicht zu Ungunsten einer vermeintlich geringeren Invasivität des Eingriffs vernachlässigt werden. Eine Plattenosteosynthese - ggf. winkelstabil verankert - sollte bei Impressionsspaltfrakturen vom Typ $41 \mathrm{~B}_{2}$ nach AO/Typ III nach Schatzker nicht in jedem Fall gemieden werden und weist insbesondere bei osteoporoseassoziierten Frakturen Vorteile hinsichtlich der postoperativen Belastbarkeit auf.

Vor allem bei den lateral nicht begrenzten und in die tiefe Beugerloge ausstrahlenden B3-Frakturen/Typ II nach Schatzker kann es rasch zu einem unkontrollierten Flüssigkeitseinstrom in Gelenkspalt und Unterschenkel kommen, hier droht u. U. ein iatrogenes Kompartimentsyn- 

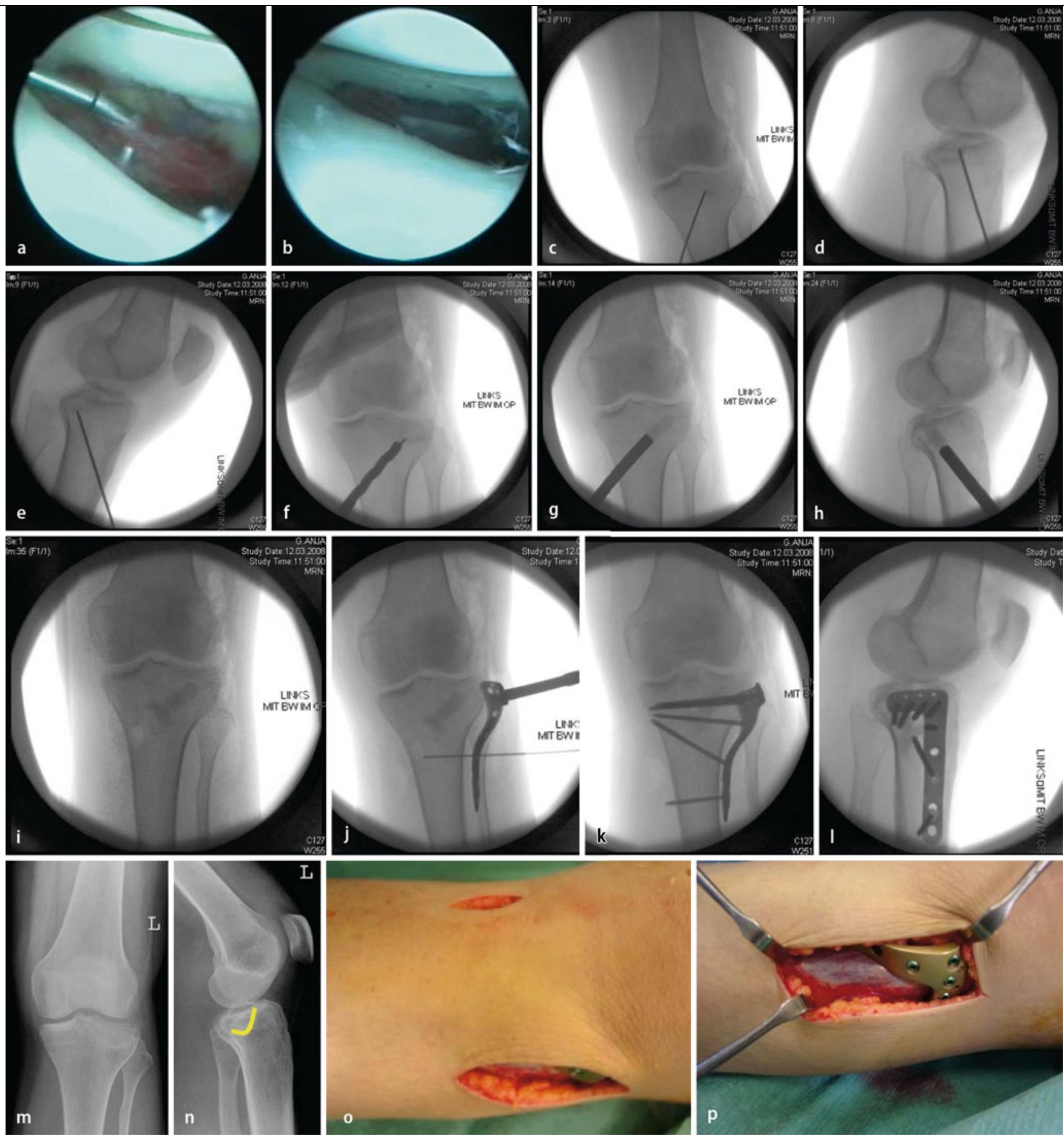

Abb. 9 \ 24-jährige Skifahrerin mit dorsolateraler Impressionsfraktur links, a hängender Außenmeniskus, in der Tiefe des Plateaus Koagel erkennbar, b nach Entfernung des Koagels Überprüfung des Meniskus auf Stabilität, erkennbare Impression, c,d BV-Verlaufskontrolle des Anzielens der Impressionszone mit Kirschner-Draht in 2 Ebenen, e Korrektur, Überbohren mit Bohrer, $\mathbf{f}$ Aufstößeln des Fragments, g,h Auffüllen der Höhle mit Endobon, i a.-p. Aufnahme, j-I Anbringen einer winkelstabilen Formplatte, $\mathbf{m}, \mathbf{n}$ Vergleich zu präoperativen Röntgenbildern, o,p intraoperative Darstellung der Weichteile und Plattenlage

drom. Daher sollte die Arthroskopie unter konstant niedrigem Pumpendruck und kontinuierlicher Absaugung erfolgen.

Bei der Versorgung von Luxationsfrakturen steht neben der Gelenkrekonstruktion u. a. die Behandlung der intraartikulären und ligamentären Begleitverlet- zungen im Vordergrund, welche durch die Arthroskopie gut sichtbar und therapierbar sind. Läsionen der Menisken (Außenmeniskus!) können arthroskopisch visualisiert werden, gleiches gilt für Rupturen des vorderen Kreuzbands und umschriebene Knorpelschäden.
Notwendige reparative Eingriffe am Kapsel-Band-Apparat werden üblicherweise offen durchgeführt, neben transossären Refixationen bietet sich die Benutzung von Knochenankern mit einhängenden Fäden an. 


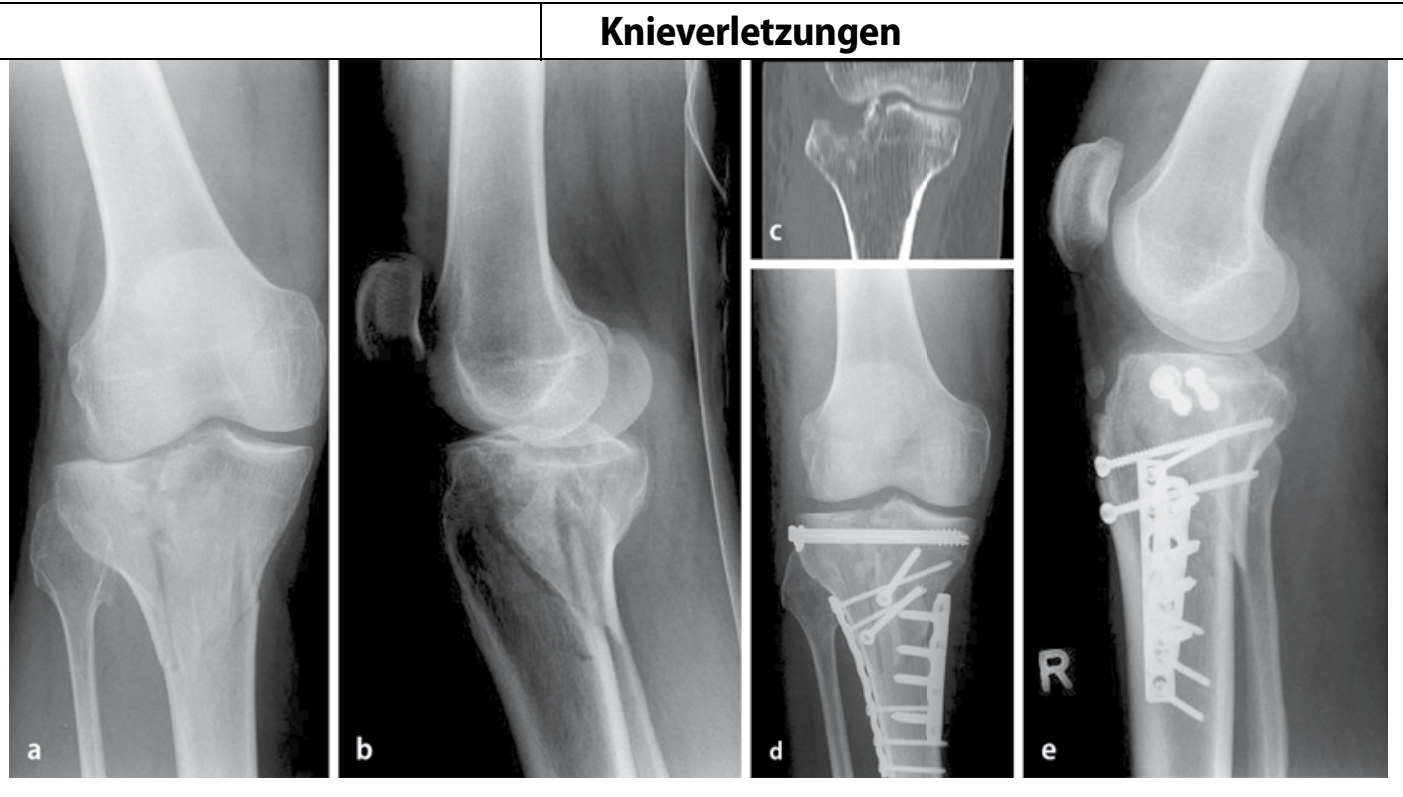

Abb. $10<$ Schienbeinkopfimpressionsfraktur mit dorsaler Depression des Plateaus, a-c vor gedeckter Reposition, a,b Röntgenbilder, c Computertomogramm, d,e postoperative Aufnahmen nach Anheben der Impression, Anheben und ventralem Verschrauben der Depression sowie Abstützen der metaphysären Frakturen beidseits mit 3,5-mm-Platten
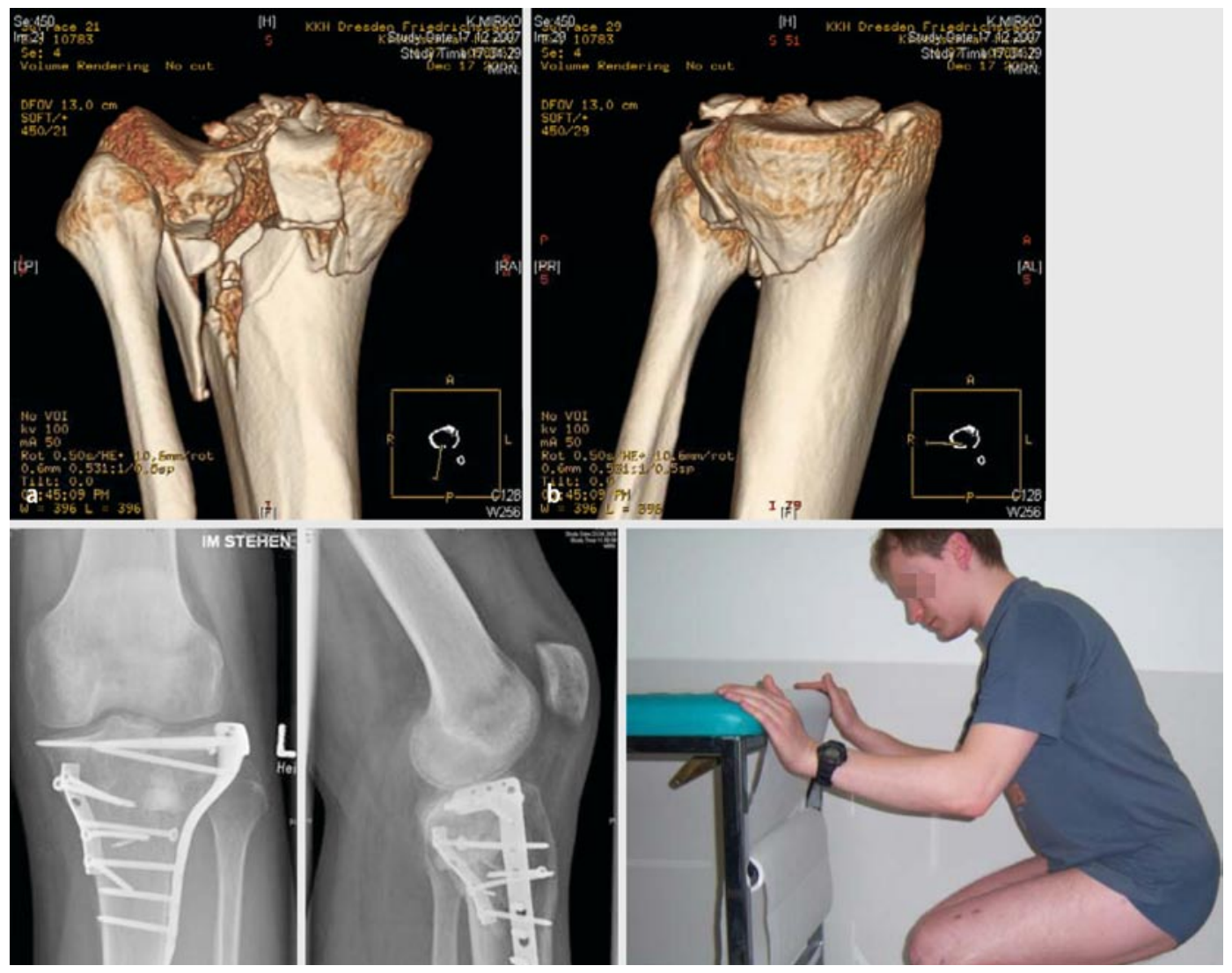

Abb. $11 \triangleleft \mathbf{a}$,b Tibiakopfluxationsfraktur (Moore V) mit ausgeprägter dorsolateraler Trümmerzone (für eine arthroskopische gestützte Operation nicht gut geeignet), c,d Operationsergebnis und e funktionelles Resultat nach bilateralem Zugehen und Plattenosteosynthese

Die Behandlung von 4-FragmentFrakturen (Schatzker Typ IV-V/Moore V) bleibt in unserer Klinik in jedem Fall der offenen Reposition und konsekutiver Osteosynthese vorbehalten, häufig durch kombinierte Zugänge von mediodorsal und lateral (• Abb. 11).

\section{Diskussion}

Wegen der gefürchteten Komplikationen wie Hautnekrose und Infekt bei der operativen Versorgung von Frakturen des Tibiakopfs über ausgedehnte Zugänge hat in den letzten Dekaden ein Trend zur Minimierung des Weichteilschadens in der Behandlung von Schienbeinkopffrakturen eingesetzt. Minimalinvasive Tech- niken, perkutane und arthroskopisch gestützte Eingriffe haben sich mehrheitlich etabliert und gehen in der Hand des erfahrenen Operateurs mit guten Ergebnissen einher $[4,6,24,28,29]$. Dennoch bleibt die Frage umstritten, welche Frakturformen von einer arthroskopisch gestützten Technik uneingeschränkt profitieren, insbesondere da die veröffentlichten Studien im Wesentlichen retrospektiv erhoben 
wurden und alle Autoren über eine vergleichsweise überschaubare Fallzahl an Eingriffen berichteten. In der Regel wurden sehr gute und gute Ergebnisse in über $90 \%$ der Fälle mitgeteilt $[1,8,10,12,13,21$, 22, 24]. Diese hervorragende Bewertung mag schon auf eine gewisse Selektion der Frakturtypen oder Patienten hinweisen.

Bezüglich arthroskopisch gestützter Repositionstechniken sind Literaturangaben zurückhaltend, da ein Vorteil gegenüber der BV-kontrollierten Reposition nicht klar nachgewiesen werden konnte $[7,16]$. Jedenfalls erscheinen uns eine optische Kontrolle der Reposition und eine mögliche Korrektur auch wegen der optischen Täuschungsmöglichkeiten der Fluorographie in Abhängigkeit von der Projektion hilfreich zu sein.

Ein uneingeschränkter Konsens findet sich bei der Feststellung, dass isolierte Frakturen der Eminentia am ehesten einer rein arthroskopisch geführten Versorgung zuzuführen sind [10, 15, 22, 25, 28]. Hier können vorteilhaft Instrumente aus der Kreuzbandchirurgie in das Therapiekonzept einbezogen werden.

Auch die isolierten Impressions- und Spaltimpressionsfrakturen $\left(41 \mathrm{~B} 1\right.$ und $-\mathrm{B}_{2}$ nach AO-Klassifikation) scheinen von einer arthroskopisch gestützten und nachfolgend perkutanen Versorgung zu profitieren. Je nach Schweregrad der Impression ist dieses operative Regime auch bei B3-Frakturen möglich, nicht selten stellen sie jedoch einen Grenzfall dar und erfordern einen Umstieg auf offene Repositions- und Fixationstechniken $[6,13,21$, 24, 28, 29].

In einem nicht unerheblichen Teil (etwa $10-25 \%)$ finden sich bei Spaltimpressions- und Luxationsfrakturen am Tibiakopf begleitende relevante Meniskusläsionen $[1,4,10,25]$. Bei Letzteren gelten folgende Therapierichtlinien: Periphere Längsrisse im Vorderhorn und in der Pars intermedia können mittels Inside-out-/Outside-in-Naht refixiert werden, im Bereich des Hinterhorns sind Allinside-Nähte gut geeignet, und komplexe Läsionen im avaskulären Anteil werden in der Regel teilreseziert [23, 25].

Einzelne Operateure favorisieren auch bei komplexeren Frakturmustern das Einbeziehen der Arthroskopie in der Versorgung von entsprechenden Typ-C-Frak- turen und schreiben diesem positive Effekte zu [4, 8, 21]. Die Mehrzahl der Autoren verwies jedoch auf die Fehler und Gefahren eines solchen Vorgehens und sah keine wirklichen Vorteile in der Versorgung bei erhöhtem Risiko für den $\mathrm{Pa}$ tienten $[6,7,10,24,25,29]$. Der vermehrte Zeit- und Personalaufwand durch die arthroskopische Technik sind hier kritisch zu hinterfragen.

Tibiakopffrakturen mit großen Trümmerzonen und weit in den Schaftbereich auslaufenden Fragmenten sind von einem Kompartimentsyndrom bedroht, im Zweifelsfall muss eine sofortige Faszienspaltung aller betroffenen Muskellogen erfolgen, für die Indikation sind v. a. klinische Gesichtspunkte entscheidend [10, 11]. Gelegentlich kann es jedoch auch im Rahmen einer arthroskopisch gestützten Versorgung ebenfalls zu einem Kompartimentsyndrom kommen, insbesondere bei den lateral nicht begrenzten und in die tiefe Beugerloge ausstrahlenden $\mathrm{B}_{3}$-Frakturen nach AO/Typ II nach Schatzker, hier ist besondere Sorgfalt zu wahren $[5,12]$.

\section{Fazit}

Die arthroskopisch gestützte Osteosynthese bei Frakturen des Schienbeinkopfs ermöglicht dem Operateur die Diagnostik von intraartikulären Begleitverletzungen, eine visuelle Darstellung der Dislokation im Tibiaplateau sowie des erreichten Ergebnisses der Reposition. Sie erlaubt ein minimalinvasives Vorgehen und stellt bei Eminentiaausrissen, Spaltund Impressionsfrakturen am Tibiakopf (A1, B1-B3 nach AO) in Verbindung mit perkutanen Osteosynthesen deshalb die Methode der Wahl dar. Die geringe Traumatisierung des Weichteilmantels wird begleitet von den Möglichkeiten, etwaige Meniskus-, Band- und Knorpelschäden gleichzeitig mit der Osteosynthese zu versorgen. Die Ergebnisse der Technik sind als durchweg gut in der Literatur mitgeteilt. Bei komplexen Brüchen und Luxationsfrakturen ist der Wert einer Arthroskopie weiterhin zu hinterfragen, stellt sie eben auch ein Gefahrenmoment dar und führt zur Verlängerung des Eingriffs.

\section{Korrespondenzadresse}

\section{Prof. Dr. F. Bonnaire}

Klinik für Unfall-Wiederherstellungsund Handchirurgie, Städtisches Klinikum, Krankenhaus Dresden Friedrichstadt Friedrichstraße 41, 01067 Dresden

bonnaire-fe@khdf.de

Interessenkonflikt. Der korrespondierende Autor gibt an, dass kein Interessenkonflikt besteht.

\section{Literatur}

1. Aitken AP (1965) Fractures of the epiphyses. Clin Orthop 41:19-23

2. Aitken AP (1965) Fractures of the proximal tibial epiphyseal cartilage. Clin Orthop 41:92-97

3. Asik M, Cetik O, Talu U, Sozen YV (2002) Arthroscopy-assisted operative management of tibial plateau fractures. Knee Surg Sports Traumatol Arthrosc 10:364-370

4. Attmanspacher W, Dittrich V, Staiger M, Stedtfeld HW (2002) Arthroskopische Rekonstruktionstechnik bei Tibiakopffrakturen. Zentralbl Chir 127:828836

5. Belanger M, Fadale $P$ (1997) Compartment syndrome of the leg after arthroscopic examination of a tibial plateau fracture. Case report and review of the literature. Arthroscopy 13(5):646-651

6. Boullion B, Schmischke D, Simanski C, Tiling T (2001) Die arthroskopisch assistierte, radiologisch kontrollierte Osteosynthese von Tibiakopffrakturen - Ergebnisse einer prospektiven Machbarkeitsstudie. Hefte Unfallchir 283:69

7. Caspari RB, Hutton PM, Whipple TL, Meyers JF (1985) The role of arthroscopy in the management of tibial plateau fractures. Arthroscopy 1:76-82

8. Chan YS, Yuan LJ, Hung SS et al (2003) Arthroscopic-assisted reduction with bilateral buttress plate fixation of complex tibial plateau fractures. Arthroscopy 19:974-984

9. Harper MC, Henstrof JE, Vessely MB et al (1995) Closed reduction and percutaneous stabilisation of tibial plateau fractures. Orthopedics 18:623-626

10. Hertel $P$ (1997) Tibiakopffrakturen. Unfallchirurg 100:508-523

11. Hohl M (1997) Tibial plateau fractures. Saunders, Philadelphia

12. Hung SS, Chao EK, Chan YS et al (2003) Arthroscopically assisted osteosynthesis for tibial plateau fractures. J Trauma 54:356-363

13. Kiefer H, Zivaljevic N, Imbriglia JE (2001) Arthroscopic reduction and internal fixation (ARIF) of lateral tibial plateau fractures. Knee Surg Sports Traumatol Arthrosc 9:167-172

14. Kutscha-Lissberg F, Dollries K, Hahn MP, Muhr G (2001) Perkutane Schraubenfixation von Schienbeinkopfbrüchen. Trauma Berufskrankh [Suppl 1] 3:54-57

15. Lemon RA, Bartlett DH (1985) Arthroscopically assisted internal fixation of certain fractures about the knee. J Trauma 25:355-358

16. Lobenhoffer P, Schulze M, Gerich T et al (1999) Closed reduction/percutaneous fixation of tibial plateau fractures: arthroscopic versus fluoroscopic control of reduction. J Orthop Trauma 13:426-431

17. Meyers MH, McKeever FM (1959) Fracture of the intercondylar eminence of the tibia. J Bone Joint Surg Am 41:209-222 
18. Müller ME, Nazarian S, Koch P, Schatzker J (1990) The comprehensive classification of fractures of long bones. Springer, Heidelberg

19. Müller ME, Allgöwer M, Schneider R (1983) Manual of internal fixation. Techniques recommended by the AO-ASIF Group. Springer, Heidelberg

20. Muhr G, Neumann K (1990) Konservative Frakturbehandlung des Tibiakopfes. Chirurg 61:767-771

21. Ohdera T, Tokunaga M, Hiroshima S et al (2003) Arthroscopic management of tibial plateau fractures - comparison with open reduction method. Arch Orthop Trauma Surg 123:489-493

22. Pino AE (1986) The role of arthroscopy in diagnosis and treatment of fractures about the knee. Orthop Trans 10:633-634

23. Petersen W, Zantop T (2006) Arthroskopische Meniskusnaht. Operat Orthop Traumatol 18:393-410

24. Pommer A, Scherenberg A, Heyde D van der, David D (2001) Arthroskopisch kontrollierte minimalinvasive Versorgung von Tibiakopffrakturen. Hefte Unfallchir 283:61

25. Raschke M, Zantop T, Petersen W (2007) Tibiakopffraktur. Chirurg 78:1157-1171

26. Salter RB, Harris WR (1963) Injuries involving the epiphyseal plate. J Joint Bone Surg Am 45-A:587622

27. Schatzker J (1987) Fractures of the tibial plateau. In: Schatzker J, Tile M (eds) The rationale of operative fracture care. Springer, Heidelberg, S 279-295

28. Scherer MA (2002) Arthroskopisch unterstützte Behandlung von Tibiakopffrakturen. Möglichkeiten und Grenzen. Trauma Berufskrankh 4:51-62

29. Stuby FM (2008) Arthroskopisch assistierte Osteosynthese bei Tibiakopffrakturen. Trauma Berufskrankh [Suppl 3] 10:366-369

30. Tscherne H, Lobenhoffer $P$ (1993) Tibial plateau fractures. Management and expected results. Clin Orthop 292: 87-100

31. Watson-Jones R (1965) Fractures and joint injuries, vol II. Churchill-Livingstone, Edinburgh 